\title{
EDITORIAL
}

\section{¿Ventilación no invasiva en la neumonía por virus N1H1?}

\section{Noninvasive ventilation in pneumonia due to N1H1virus}

\author{
M.L. Gómez Grande
}

\author{
Unidad de Cuidados Intensivos, Hospital General Universitario, Ciudad Real
}

Recibido el 16 de mayo de 2011; aceptado el 20 de mayo de 2011

Durante el 2009, las unidades de cuidados intensivos asistieron a los pacientes afectados por la pandemia de gripe ocasionada por el virus $\mathrm{N} 1 \mathrm{H} 1$ en estado crítico $^{1}$; posteriormente hemos atendido a los pacientes de la primera epidemia en las últimas semanas de 2010 y primeras de $2011^{2}$ aplicando las medidas aprendidas durante la pandemia. La característica común de estos pacientes es una grave afectación respiratoria y desarrollo del síndrome del distrés respiratorio agudo (SDRA) marcado por la hipoxemia grave y la necesidad de aplicar estrategias ventilatorias y tratamientos de rescate (decúbito prono, oxido nítrico, oxigenación extracorpórea, etc. $)^{3,4}$ para corregir y superar la situación. Se ha asociado a peor pronóstico la presencia de disfunción multiorgánica, necesidad de ventilación mecánica $(V M)^{5}$, fracaso renal agudo ${ }^{6}$, coinfección ${ }^{7}$, retraso en la administración del antiviral oseltamivir ${ }^{8}$ y la obesidad ${ }^{9}$ que junto con la coinfección ${ }^{7}$ se han asociado a un aumento de consumo de recursos; además el embarazo se asoció con un aumento del riesgo de desarrollar neumonía viral primaria ${ }^{10}$. En la epidemia de 2011, la enfermedad hematológica, la gravedad general, los infiltrados en la radiografía de tórax y la necesidad de VM fueron las variables asociadas de forma independiente con la mortalidad ${ }^{2}$.

La SEMICYUC elaboró unas recomendaciones de manejo durante la fase de pandemia ${ }^{11}$. Dentro de ellas las referentes a la utilización de ventilación no invasiva (VNI) han seguido vigentes en la epidemia del 2010-2011: En los pacientes que requieran soporte respiratorio y en los que exista una alta sospecha de infección por el nuevo virus de la gripe $A(H 1 N 1) v$ no es recomendable utilizar ventilación no inva-

Correo electrónico: mlgomezgrande2000@yahoo.es siva, debido al riesgo de generación de aerosoles, que aumentan el riesgo de transmisión al personal sanitario, así como a sus pobres resultados clínicos en este tipo de pacientes. Si se decide utilizar VNI se deberá evaluar la relación entre riesgo y beneficio, considerando especialmente:

a. Reservar esta modalidad ventilatoria para pacientes sin criterios de SDRA

b. Realizar la VNI preferentemente en habitaciones con presión negativa

c. Utilizar preferentemente respiradores con doble circuito

d. Utilizar los accesorios para la vía aérea con mayor seguridad (por ejemplo, mascarillas que cubran toda la cara)

e. Cumplir estrictamente con todas las medidas de protección para el personal (aislamiento por aire)

f. Nunca realizar VNI en Urgencias ni en habitaciones o salas compartidas.

Asimismo, la European Respiratory Society y la European Society of Intensive Care Medicine ${ }^{12}$ recomiendan evitar la VNI e intubar a los pacientes con infección por el nuevo virus $\mathrm{H} 1 \mathrm{~N} 1$ ingresados en las unidades de cuidados intensivos con hipoxemia grave, rápido desarrollo de SDRA, fracaso multiorgánico e hipoxia refractaria; en su ausencia, la VNI debe ser considerada únicamente en pacientes con fallo respiratorio agudo hipercápnico moderado secundario a una exacerbación de un trastorno respiratorio crónico, insuficiencia respiratoria aguda secundaria a edema agudo de pulmón y fallo respiratorio postextubación tras SDRA secundarios a infección por virus H1N1.

Durante la pandemia se comunicó la utilización de la VNI en un $25-45 \%$ de los pacientes, con un $75 \%$ de fracaso ${ }^{13-15}$ los pacientes que fracasaron presentaron valores en las escalas 
de gravedad mayores y posiblemente debieron ser intubados desde el inicio del cuadro ${ }^{13}$. En la primera epidemia 2010-2011 en el boletín informativo del GTEI de la SEMICYUC $n^{\circ} 2$, el $80,3 \%(n=237)$ de los pacientes requirieron VM. De ellos el $35 \%$ recibieron VMNI inicial y un 50\% de los mismos fracasaron en esa modalidad y debieron ser intubados posteriormente $[\ldots]^{16}$ mejorando los resultados de la pandemia, de forma probablemente secundario a la implementación de las medidas con la experiencia adquirida, similares a los datos obtenidos en metaanálisis que analizan la utilización de VNI en los pacientes con SDRA/ALI ${ }^{17}$.

La VNI se ha utilizado con éxito en los pacientes con insuficiencia respiratoria aguda secundaria a edema agudo de pulmón, y en la insuficiencia respiratoria aguda hipercápnica (nivel de evidencia IA), sin embargo, en la insuficiencia respiratoria aguda hipoxémica existe controversia, debiendo el clínico individualizar cada caso. Una contraindicación relativa para utilizar la VNI en la insuficiencia respiratoria aguda hipoxémica es la presencia de un cociente $\mathrm{PO}_{2} / \mathrm{FiO}_{2}<100^{18}$, sin embargo, de los pacientes del registro de Belenguer et $\mathrm{al}^{19}$ con $\mathrm{PO}_{2} / \mathrm{FiO}_{2}$ de ingreso, 87 presentan parámetros de buen pronóstico en la primera hora, definidos en la literatura ${ }^{18}$. Incluso en las series presentadas la presencia de coinfección junto a la neumonía viral presentó peor pronóstico ${ }^{7}$, ausente en la serie de Berenguer et $\mathrm{al}^{19}$.

Los buenos resultados presentados por Belenguer et $\mathrm{al}^{19}$ pueden justificarse por: 1) la afectación respiratoria exclusiva con ausencia de disfunción de otros órganos, lo que asocia APACHE II y bajos SOFA, junto a la ausencia de enfermedad de base y de coinfección; 2) la experiencia del equipo está comunicada ${ }^{20-23}$ como fundamental para obtener el éxito en la aplicación de la VNI; y 3) un riguroso sistema de aislamiento siguiendo las recomendaciones de la SEMICYUC ${ }^{11}$ y el servicio de medicina preventiva local.

Es posible que la experiencia sumada a la evidencia consigan definir a aquellos pacientes que podrían beneficiarse de estrategias menos agresivas para conseguir la resolución de su proceso. La VNI durante la insuficiencia respiratoria aguda hipoxémica secundaria a neumonía aguda por virus $\mathrm{N} 1 \mathrm{H} 1$ debería aplicarse únicamente a pacientes muy seleccionados y siempre por un equipo de gran experiencia, sumado a todas las medidas terapéuticas disponibles definidas como eficaces.

\section{Bibliografía}

1. Rodríguez A, Socías L, Guerrero JE, Figueira JC, González N, Maravi Pomar E, et al., Grupo Español de Trabajo de Gripe A Grave/Sociedad Española de Medicina Intensiva, Crítica y Unidades Coronarias. Gripe A pandémica en Cuidados Intensivos: experiencia en España y Latinoamérica. Med Intensiva. 2010;34:87-94.

2. Rodríguez A, Martín-Loeches I, Bonastre J, Olaechea P, Álvarez-Lerma F, Zaragoza R, et al., SEMICYUC-CIBERES-REIPI Working Group. First influenza season after the 2009 pandemic influenza: report of the first 300 ICU admissions in Spain. Med Intensiva. 2011;35:208-16.

3. Mozo-Martín T, Gordo-Vidal F. Estrategias frente a la hipoxemia refractaria en los casos de gripe A grave. Med Intensiva. 2010;34:431-4.

4. Napolitano LM, Park PK, Raghavendran K, Bartlett RH. Nonventilatory strategies for patients with life-threatening $2009 \mathrm{H} 1 \mathrm{~N} 1$ influenza and severe respiratory failure. Crit Care Med. 2010;38 Suppl:e74-90.

5. Martín-Loeches I, Rodríguez A, Bonastre J, Zaragoza R, Sierra R, Marqués A, et al., the H1N1 SEMICYUC Working Group. Severe pandemic (H1N1)v influenza A infection: Report on the first deaths in Spain. Respirology. 2011;16:78-85.

6. Martín-Loeches I, Papiol E, Rodríguez A, Díaz E, Zaragoza R, Granada R, et al., H1N1 SEMICYUC Working Group. Acute kidney injury in critical ill patients affected by influenza A (H1N1) v Infection. Critical Care. 2011;15:R66.

7. Martín-Loeches I, Sánchez-Corral A, Díaz E, Granada R, Zaragoza R, Villavicencio C, et al., and H1N1 SEMICYUC Working Group. Community-acquired respiratory coinfection in critically ill patients with pandemic 2009 influenza $A(H 1 N 1)$ virus. Chest. 2011;139:555-62.

8. Rodríguez A, Díaz E, Martín-Loeches I, Sandiumenge A, Canadell L, Díaz JJ, et al., on behalf of the N1H1 SEMICYUC Working Group. Impact of early oseltamivir treatment on outcome in critically ill patients with 2009 pandemic influenza A. J Antimicrob Chemother. 2011;66:1140-9.

9. Díaz E, Rodríguez A, Martín-Loeches I, Lorente L, Martín MM, Pozo JC, et al., H1N1 SEMICYUC Working Group. Impact of obesity in patients infected with 2009 influenza A(H1N1). Chest. 2011;139:382-6.

10. Maraví-Poma E, Martín-Loeches I, Regidor E, Laplaza C, Cambra K, Aldunate S, et al. Grupo Español de trabajo de Gripe Grave A (SEMICYUC). Severe 2009 H1N1 influenza in pregnant women in Spain. Crit Care Med. 2011;39:945-51.

11. Rodríguez A, Gordo F. Diagnóstico y tratamiento de los pacientes adultos con insuficiencia respiratoria aguda grave por el nuevo virus de la gripe A (H1N1). SEMICYUC. Disponible en: http://www.semicyuc.org/sites/default/files/protocolo_ manejo_20091015.pdf.

12. Conti G, Larrsson A, Nava S, Navalesi P. On the role of non-invasive ventilation to treat patients during the H1N1 influenza pandemic. Disponible en: http://dev.ersnet.org/uploads/Document/63/WEB_CHEMIN_ 5410_1258624143.pdf.

13. Rello J, Rodríguez A, Ibáñez P, Socias L, Cebrián J, Marques A, et al., for the H1N1 SEMICYUC Working Group. Intensive care adult patients with severe respiratory failure caused by influenza A (H1N1) v in Spain. Crit Care. 2009;13:R148.

14. Kumar A, Zarychanski R, Pinto R, Cook DJ, Marshal J, Lacroix J, et al. Critically ill patients with 2009 influenza A/H1N1 infection in Canada. JAMA. 2009;361:1925-34.

15. Nin N, Soto L, Hurtado J, Lorente JA, Buroni M, Arancibia F, et al. Clinical characteristics and outcomes of patients with 2009 influenza $\mathrm{A}(\mathrm{H} 1 \mathrm{~N} 1)$ virus infection with respiratory failure requiring mechanical ventilation. J Crit Care. 2011;26:186-92. Epub 2010 Aug 4.

16. Boletín Informativo $N^{\circ} 2(11 / 02 / 2011)$. Grupo Español de Trabajo Gripe A Grave. GTEl. Disponible en: www.semicyuc.org/sites/default/files/newsletter_na_2.pdf.

17. Antonelli M, Conti G, Esquinas A, Montini L, Maggiore SM, Bello $G$, et al. A multiple-center survey on the use in clinical practice of noninvasive ventilation as a first-line intervention for acute respiratory distress syndrome. Crit Care Med. 2007;35: 18-25.

18. Agarwal R, Aggarwal AN, Gupta D. Role of noninvasive ventilation in acute lung injury/acute respiratory distress syndrome: a proportion meta-analysis. Respir Care. 2010;55:1653-60.

19. Belenguer Muncharaz A, Reig-Valero R, Altaba-Tena S, CaseroRoig P, Ferrándiz-Selles A. Utilización de la ventilación no invasiva en la neumonía grave por virus H1N1. Med Intensiva. 2011;35:470-7.

20. Sinuff T, Kahnamoui K, Cook DJ, Giacomini M. Practice guidelines as multipurpose tools: a qualitative study of noninvasive ventilation. Crit Care Med. 2007;35:776-82. 
21. Maheshwari V, Paioli D, Rothaar R, Hill NS. Utilization of noninvasive ventilation in acute care hospitals: a regional survey. Chest. 2006;129:1226-33.

22. Meduri GU, Turner RE, Abou-Shala N, Wunderink R, Tolley E. Noninvasive positive pressure ventilation via face mask.
First-line intervention in patients with acute hypercapnic and hypoxemic respiratory failure. Chest. 1996;109: 179-93.

23. Nava S, Ceriana P. Causes of failure of noninvasive mechanical ventilation. Respir Care. 2004;49:295-303. 